\title{
ERRATUM
}

\section{Erratum to: New Therapeutic Approaches for Management of Sport-Induced Muscle Strains}

Angelo De Carli · Piero Volpi · Iva Pelosini · Andrea Ferrretti · Gianluca Melegati · Luigi Mossa ·

Davide Tornese · Laura de Girolamo · Carmelo Scarpignato

(C) 2010 Springer Healthcare

Erratum to: De Carli A, et al. New therapeutic approaches for management of sport-induced muscle strains. Adv Ther. 2009;26(12):1072-1083.

The publishers would like to add an erratum, as suggested by the authors Angelo De Carli, et al., to remove any ambiguity that may have been caused by the use of the term "relative risk" in Table 1 (shown below). As such, the authors would like to replace the term with "relative benefit."

The original table header is: "Relative risk (fixed) and number needed to treat (NNT) from a metaanalysis of various topically applied nonsteroidal anti-inflammatory drugs (NSAIDs) compared with placebo for the treatment of acute musculoskeletal pain conditions."

The rephrased table header should read: "Relative benefit (fixed) and number needed to treat (NNT) from a meta-analysis of various topically applied nonsteroidal anti-inflammatory drugs (NSAIDs) compared with placebo for the treatment of acute musculoskeletal pain conditions." Here, relative benefit is defined as the relative risk of experiencing a 50\% reduction in pain in the active drug group versus placebo.

The revised table with header is shown below, for clarity.

Table 1. Relative benefit (fixed) and number needed to treat (NNT) from a meta-analysis of various topically applied nonsteroidal anti-inflammatory drugs (NSAIDs) compared with placebo for the treatment of acute musculoskeletal pain conditions.

\begin{tabular}{lcc}
\hline & $\begin{array}{c}\text { Relative benefit* } \\
(\mathbf{9 5 \%} \text { CI })\end{array}$ & $\begin{array}{c}\text { NNT } \\
(\mathbf{9 5 \%} \text { CI })\end{array}$ \\
\hline Ketoprofen & $2.01(1.71,2.37)$ & $2.6(2.2,3.3)$ \\
Ibuprofen & $1.69(1.35,2.12)$ & $4.1(2.9,6.9)$ \\
Piroxicam & $1.54(1.29,1.84)$ & $4.7(3.4,7.7)$ \\
Indomethacin & $1.30(0.98,1.72)$ & $10.0(5.2, \infty)$ \\
\hline
\end{tabular}

${ }^{*}$ Relative benefit is defined as the relative risk of experiencing a $50 \%$ reduction in pain in the active drug group versus placebo. 